\title{
Antithyroid Peroxidase Antibodies in Multinodular Hashimoto's Thyroiditis Indicate a Variant Etiology
}

\author{
Pabithadevi B. Mehanathan $\mathbb{D}^{\mathbb{D}},{ }^{1}$ R. Raskin Erusan, ${ }^{2}$ K. Shantaraman ${ }^{\mathbb{D}},{ }^{3}$ and S. M. Kannan ${ }^{4}$ \\ ${ }^{1}$ General Surgery, Tirunelveli Medical College, Tirunelveli, India \\ ${ }^{2}$ Multi Disciplinary Research Unit, Tirunelveli Medical College, Tirunelveli, India \\ ${ }^{3}$ Department of Pathology, Tirunelveli Medical College, Tirunelveli, India \\ ${ }^{4}$ Tirunelveli Medical College, Tirunelveli, India
}

Correspondence should be addressed to K. Shantaraman; shantaraman.kal@gmail.com

Received 17 February 2019; Accepted 20 May 2019; Published 21 July 2019

Academic Editor: Noriyuki Koibuchi

Copyright (C) 2019 Pabithadevi B. Mehanathan et al. This is an open access article distributed under the Creative Commons Attribution License, which permits unrestricted use, distribution, and reproduction in any medium, provided the original work is properly cited.

\begin{abstract}
Introduction. Hashimoto's thyroiditis (HT) is a common autoimmune thyroid disorder, which predominantly presents as a diffuse goiter, with few studies which report HT presenting as multinodular goiter, with variable frequencies ranging from $59 \%$ to $78.6 \%$ especially from south Indian populations. This variant clinical presentation may have diagnostic challenges which require further analysis. Anti-TPO antibodies are more common (90-95\%) in Hashimoto's thyroiditis than anti-TG antibodies in Hashimoto's thyroiditis. This study analyzes the clinical features and the correlation of anti-TPO levels with diffuse and multinodular forms of HT. Material and Methods. This study was conducted in the Department of General Surgery in a tertiary care hospital in south Tamil Nadu. Patients presenting with clinical features of a thyroid disorder were interviewed and given a detailed clinical, radiological examination and guided FNAC. Those patients diagnosed by FNAC as HT were registered and a sample of $3 c c$ of blood was drawn for T3, T4, TSH, and anti-TPO analysis. All the data were tabulated. Results and Discussion. Of the 212 patients who presented with goiters, 96 were diagnosed by FNAC as having a cytological picture suggestive of Hashimoto's thyroiditis. Of these 96 patients with HT, 46 (47.9\%) were multinodular (HT-MNG), 14 (14.58\%) were solitary nodules (HT-SNT), and the remaining 36 (37.5\%) were diffuse goiters (HT-D). Of the 46 patients who are HT-MNG, 36.9\% had elevated anti-TPO-Ab (more than 35.0U/1) and 63.1\% had normal/lower values (less than 35.0U/1). But of 36 patients with HT-D, 77.7\% had elevated anti-TPO-Ab levels ( $>35 \mathrm{U} / \mathrm{l})$. Chi square statistics was 15.8346 and the p value is 0.0005 (<.05). Eight cases of HT-D and 3 cases of HT-MNG had hyperthyroidism and 3 cases of HT-D had hypothyroidism and all other cases were in euthyroid state. Conclusion. Patients presenting as multinodular Hashimoto's thyroiditis have low prevalence of elevated anti-TPO-Ab than diffuse HT which suggests that multinodular form of Hashimoto's thyroiditis is a unique clinical entity with etiopathogenesis that is at variance with the diffuse form.
\end{abstract}

\section{Background}

Hashimoto's thyroiditis (HT) is a common autoimmune thyroid disorder, characterised by follicular lymphocytic infiltration in the thyroid gland with formation of germinal centers, Hurthle cell change, atrophy of the follicular epithelial cells, and gradual fibrous replacement of the thyroid parenchyma. HT predominantly presents as a diffuse goiter [1], with few reports of a presentation as a multinodular goiter [2]. These multinodular HT patients are reported with variable frequencies ranging from $59 \%$ to $78.6 \%$ especially from south
Indian populations [3]. This variant clinical presentation may have diagnostic challenges which require further analysis.

The destruction of thyroid cells in HT is associated with various cellular and antibody mediated immune processes which include thyroid autoantibodies (TAbs) against thyroid peroxidase (TPO) and thyroglobulin (Tg). Anti-TPO antibodies are more common than anti-Tg antibodies and more indicative for thyroid disease [4] and are detected in $90-95 \%$ of AITD patients, $80 \%$ of GD, and $10-15 \%$ of non-AITD patients [5]. While anti-TPO antibodies may act cytotoxic on thyrocytes in HT they do not have an 
established role in GD [6]. Exact values cannot be compared directly since sensitivities of the assays differ, but the range of $>35 \mathrm{U} / \mathrm{l}$ for anti-TPO, according to most laboratories can serve as approximate indication for the diagnosis of HT [7]. Various studies reported a high occurrence $(61 \%)$ of Hashimoto's thyroiditis in the form of diffuse goiter and 93 percent of these patients were anti-TPO positive [8]. Similar studies on multinodular type of HT are sparse and hence its etiopathogenesis requires to be documented. This study analyzes the clinical features and the correlation of anti-TPO levels with diffuse and multinodular forms of HT.

\section{Methodology}

This study was conducted in the department of General Surgery in a tertiary care hospital in south Tamil Nadu after obtaining the institutional ethics committee permission. Patients presenting in the outpatient department with visible thyroid enlargement or with clinical features indicative of a thyroid disorder were interviewed and given a detailed clinical examination, which was followed by radiological examination with ultra sonogram, biochemical tests for thyroid hormones, and guided fine needle aspiration cytology (FNAC). The patients were diagnosed to have Hashimoto's thyroiditis by FNAC based on the presence of oxyphilic (Hurthle) cells, lymphocytes, few plasma cells, and the presence of moderate to scant amount of colloid in the background.

These patients diagnosed by FNAC as HT were registered and a sample of $3 \mathrm{cc}$ of blood was drawn for the thyroid analysis (T3, T4, TSH, and anti-TPO) which were performed using chemiluminescence immunoassay (Beckman \& Coulter Inc.) using Access II kits and calibrators after obtaining a written informed consent. These patients were then prescribed medical and surgical management as per the standard protocols, and thyroidectomy was performed when indicated. The thyroidectomy samples were sent for histopathological examination and only those patients diagnosed as Hashimoto's thyroiditis in histology were included for final analysis in the study.

The patients with histological diagnosis of HT were classified as those presenting as diffuse goiter (HT-D), solitary nodule of thyroid (HT-SNT), or multinodular goiter (HTMNG) based on the clinical and radiological evidences. A structured proforma was used to document the data of clinical history, present and past morbidities, and socioeconomic, demographic, clinical, radiological, cytopathological, and biochemical parameters including serum T3, T4, TSH, and anti-TPO antibody. The results were tabulated and analyzed using a chi square test.

\section{Results}

Of the 212 patients who presented with goiters, 96 were diagnosed by FNAC as having a cytological picture suggestive of Hashimoto's thyroiditis. Of these 96 patients, 91 underwent surgical management with hemi/subtotal thyroidectomy. The tissue samples of all these 91 patients presented with histological features confirmatory of HT including dense lymphocytic infiltrates forming follicles with reactive germinal centers and Hurthle cell change of the follicular epithelial cells. Of these 96 patients with HT, 46 (47.9\%) were multinodular (HT-MNG), 14 (14.58\%) were solitary nodules (HT-SNT), and the remaining 36 (37.5\%) were diffuse goiters (HT-D).

The assays for T3, T4, TSH, and anti-TPO antibody are tabulated with the clinical information. Of the 46 patients who are HT-MNG, 17 had elevated anti-TPO-Ab (more than $35.0 \mathrm{U} / \mathrm{l}$ ), and $29 \mathrm{had}$ normal/lower values of less than $35.0 \mathrm{U} / \mathrm{l}$. Of 36 patients with HT-D, 28 had anti-TPO-Ab levels $>35 \mathrm{U} / 1$ and 8 patients had anti-TPO-Ab levels $<35 \mathrm{U} / 1$ (Table 1). Chi square statistics was 15.8346 and the $\mathrm{p}$ value is $0.0005(<.05)$. Eight cases of HT-D and 3 cases of HT-MNG had hyperthyroidism and 3 cases of HT-D had hypothyroidism and all other cases were in euthyroid state.

\section{Discussion}

Hashimoto disease is a complex autoimmune disease, more prevalent among elderly women [9], and in geographic regions which are iodine sufficient (e.g., USA) or iodine excess (e.g., Japan) [10]. Thyroid peroxidase is a transmembrane protein located in the apical membrane of thyroid follicular cells, involved in the synthesis of thyroid hormones. Thyroid microsomal antibodies, namely, anti-TPO antibody, is a thyroid autoantibody targeting thyroid peroxidases [11] and is considered diagnostic of both Grave's disease and Hashimoto's thyroiditis, while in Hashimoto's thyroiditis, anti-TPO antibody is reported in approximately $90 \%$ of patients [12].

Our study conducted in south Tamil Nadu has observed that $47.9 \%$ of our patients with Hashimoto's thyroiditis were having multinodular thyroid, while $14.5 \%$ were solitary nodules and $37.5 \%$ were diffuse forms. Of the HT patients with diffuse thyroid enlargement, $77.7 \%$ had elevated titres of anti-TPO-Ab, while $36.9 \%$ of our patients with multinodular goiters had elevated titres of anti-TPO-Ab and 63\% had low titres of anti-TPO-Ab. But the histological patterns of multinodular and diffuse forms of HT were not different.

Two studies reported from southern India, Tina Thomas et al. (2014), reported that $61 \%$ of Hashimoto's thyroiditis presented as diffuse goiter with $93 \%$ of them positive for antiTPO-Ab [8], while Anila KR (2016) reported that nodular form of HT was seen early in the progression of disease, associated with normal TSH and high anti-TPO levels, which became predominantly diffuse later [13]. Shirish.S.C et al. (2014) reported that $67.30 \%$ of their patients had diffuse goiters, while $30.76 \%$ had uneven enlargement and $1.92 \%$ had solitary nodule, while serum anti-TPO-Ab were elevated in $61.52 \%$ patients [14]. These studies are at variance with our observation that nodular forms of HT were associated with higher incidence of high titres of anti-TPO-Ab, while we observed in the converse that it was lesser in our patients. We have also reported the analysis of clinical data of Hashimoto's thyroiditis patients for a period of 3 years (20132015) which indicated a predominance of multinodular HT with euthyroid state in this region at variance with western literature [3]. 
TABLE 1: Distribution of HT and relation with thyroid hormone status and anti-TPO Ab.

\begin{tabular}{|c|c|c|c|c|c|c|}
\hline No & Parameter & & HT-D & HT-SN & HT-MN & Total \\
\hline 1 & No. of Patients (Percentage) & & $36(37.5)$ & $14(14.58)$ & $46(47.92)$ & 96 \\
\hline 2 & Mean Age in years & & 41.6 & 44 & 43 & 42.87 \\
\hline \multirow[t]{2}{*}{3} & Gender & Male & 1 & 1 & 0 & 2 \\
\hline & & Female & 35 & 13 & 46 & 94 \\
\hline \multirow[t]{3}{*}{4} & Presenting Features & Goitre & 35 & 14 & 46 & 96 \\
\hline & & Hypothyroidism & 0 & 0 & 0 & 0 \\
\hline & & Hyperthyroidism & 1 & 0 & 0 & 1 \\
\hline \multirow[t]{15}{*}{5} & Biochemical Tests & & & & & \\
\hline & Serum T3 & High & 8 & 0 & 3 & 11 \\
\hline & (Ref Range: 60-200 ng/ml) & Normal & 25 & 14 & 43 & 82 \\
\hline & & Low & 3 & 0 & 0 & 3 \\
\hline & Serum T4 & High & 8 & 0 & 3 & 11 \\
\hline & (Ref Range: $4.5-12 \mathrm{ug} / \mathrm{ml}$ ) & Normal & 25 & 14 & 43 & 82 \\
\hline & & Low & 3 & 0 & 0 & 3 \\
\hline & Serum TSH & High & 3 & 0 & 0 & 3 \\
\hline & (Ref Range: 0.3-5 uIU/ml) & Normal & 25 & 14 & 43 & 82 \\
\hline & & Low & 8 & 0 & 3 & 11 \\
\hline & Serum Anti-TPOAb & $<35$ & 8 & 10 & 29 & 47 \\
\hline & (Ref Range: 0.25-35 U/l) & $36-200$ & 4 & 0 & 6 & 10 \\
\hline & & $201-500$ & 5 & 2 & 3 & 10 \\
\hline & & $501-976$ & 8 & 2 & 4 & 14 \\
\hline & & $>976$ & 11 & 0 & 4 & 15 \\
\hline
\end{tabular}

Note. HT: Hashimoto's thyroiditis, HT-D: diffuse type, HT-SN: solitary nodular type, and HT-MN: multinodular type.

In routine, Hashimoto's thyroiditis is diagnosed by the elevated level of anti-TPO-Ab and high level of TSH and treated with levothyroxine, and many studies [15] have shown that the serum levels of anti-TPO-Ab decline during levothyroxine treatment. But in our patients with multinodular form of HT with low titre of anti-TPO-Ab, the established conservative line of treatment is ineffective.

Several population-based studies [16-18] have reported higher prevalence of anti-TPO antibody in regions with higher iodine consumption, namely, $18 \%$ in iodine sufficient areas [19] and 25\% in areas with excessive iodine intake [20], while that was approximately $13 \%$ in iodine deficiency areas [16]. There are reports of almost fourfold increase in anti-TPO positive HT after exposure to higher iodine levels consequent to the iodine prophylaxis in iodine deficient areas [19-21]. Katja Zaletel et al. (2011) have reported [22] that deliberate exposure to $500 \mu \mathrm{g}$ of iodine provoked thyroid autoimmunity synthesis in $20 \%$ of previously healthy individuals [23]. In a study conducted at Denmark also reports that the prevalence of TPO-Ab before and after mandatory iodization of salt was $14.3 \%$ and $23.8 \%$, respectively [24]. The familial clustering of autoimmune thyroid disease and the presence of anti-TPO$\mathrm{Ab}$ in $16.7 \%$ to $60 \%$ of first-degree relatives of patients has been reported by several studies [25-29]. Among first-degree relatives of patients with HT, $34 \%$ were demonstrated to be positive for anti-TPO-Ab compared to $13 \%$ among persons not related [29]. The sibling risk ratio for HT, calculated on the basis of the data from the NHANES III study, was 28 , confirming a significant interaction of genetic factors in disease development [30]. In Hashimoto's thyroiditis, however a small subset of patients (10\%) are reported with clinically evident disease, and a negative serum anti-TPO-Ab [31].

This study suggests that multinodular form of Hashimoto's thyroiditis is a unique clinical entity with etiopathogenesis that is at variance with the diffuse form, with possible genetic variations and differences of metabolic and environmental factors. Since our sample size is small, the preposition of multinodular HT as a unique clinical entity with specific etiopathogenesis needs further study.

\section{Conclusion}

Multinodular form of Hashimoto's thyroiditis is a unique clinical entity with possible specific etiology and pathogenesis, different from the established etiopathogenesis attributed to the diffuse form of HT.

\section{Data Availability}

The data used to support the findings of this study are available from the corresponding author upon request.

\section{Disclosure}

The authors alone are responsible for the content and writings of this article. 


\section{Conflicts of Interest}

The authors report no conflicts of interest.

\section{Acknowledgments}

We acknowledge the Department of Health Research, Government of India, for the support and funding for this project under the Multi Disciplinary Research Unit, Tirunelveli Medical College, Tirunelveli.

\section{References}

[1] T. Thomas, S. Sreedharan, U. N. Khadilkar et al., "Clinical, biochemical \& cytomorphologic study on hashimoto's thyroiditis," Indian Journal of Medical Research, vol. 140, pp. 729-735, 2014.

[2] P. R. K. Bhargav and S. Shekher, "Surgical indications for goiter with background Hashimoto's thyroiditis an institutional experience," Indian Journal of Surgery, vol. 73, no. 6, pp. 414-418, 2011.

[3] B. Pabithadevi and K. Shantaraman, "Hashimoto's thyroiditis: a predominantly multinodular thyroid disorder in coastal South India," Annals of Pathology and Laboratory Medicine, vol. 5, no. 3, pp. 204-208, 2018.

[4] F. S. Balucan, S. A. Morshed, and T. F. Davies, "Thyroid autoantibodies in pregnancy: their role, regulation and clinical relevance," Journal of Thyroid Research, vol. 2013, Article ID 182472, 15 pages, 2013.

[5] G. de Carvalho, C. L. Perez, and L. S. Ward, "The clinical use of thyroid function tests," Arquivos Brasileiros de Endocrinologia \& Metabologia, vol. 57, no. 3, pp. 193-204, 2013.

[6] L. J. DeGroot, "Graves' disease and the manifestations of thyrotoxicosis," in Endotext, L. J. De Groot, G. Chrousos, K. Dungan et al., Eds., MDText.Com, Inc., South Dartmouth, MA, USA, 2000.

[7] G. Elhomsy, "Antithyroid Antibody," in Laboratory Medicine, E. Staros, Ed., 2014.

[8] T. Thomas, S. Sreedharan, U. N. Khadilkar et al., "Clinical, biochemical \& cytomorphologic study on hashimoto's thyroiditis," Indian Journal of Medical Research, vol. 140, no. 6, pp. 729-735, 2014.

[9] C. A. Spencer, J. G. Hollowell, M. Kazarosyan, and L. E. Braverman, "National Health and Nutrition Examination Survey III Thyroid-Stimulating Hormone (TSH)-thyroperoxidase antibody relationships demonstrate that TSH upper reference limits may be skewed by occult thyroid dysfunction," The Journal of Clinical Endocrinology \& Metabolism, vol. 92, no. 11, pp. 4236-4240, 2007.

[10] Y. H. Dong and D. G. Fu, "Autoimmune thyroid disease: mechanism, genetics and current knowledge," European Review for Medical and Pharmacological Sciences, vol. 18, no. 23, pp. 3611-3618, 2014.

[11] B. Czarnocka, J. Ruf, M. Ferrand, P. Carayon, and S. Lissitzky, "Purification of the human thyroid peroxidase and its identification as the microsomal antigen involved in autoimmune thyroid diseases," FEBS Letters, vol. 190, no. 1, pp. 147-152, 1985.

[12] S. Mariotti, P. Caturegli, P. Piccolo, G. Barbesino, and A. Pinchera, "Antithyroid peroxidase autoantibodies in thyroid disease," The Journal of Clinical Endocrinology \& Metabolism, vol. 71, no. 3, pp. 661-669, 1990.
[13] K. Anila, N. Nayak, and K. Jayasree, "Cytomorphologic spectrum of lymphocytic thyroiditis and correlation between cytological grading and biochemical parameters," Journal of Cytology, vol. 33, no. 3, pp. 145-149, 2016.

[14] S. Chandanwale, C. Gore, S. Bamanikar, N. Gupta, and K. Gupta, "Cytomorphologic spectrum of Hashimoto's thyroiditis and its clinical correlation: a retrospective study of 52 patients," CytoJournal, vol. 11, no. 1, article no. 9, 2014.

[15] M. Schmidt, M. Voell, I. Rahlff et al., "Long-term follow-up of antithyroid peroxidase antibodies in patients with chronic autoimmune thyroiditis (Hashimoto's thyroiditis) treated with levothyroxine," Thyroid, vol. 18, no. 7, pp. 755-760, 2008.

[16] F. Aghini-Lombardi, L. Antonangeli, E. Martino et al., "The spectrum of thyroid disorders in an iodine-deficient community: the pescopagano survey," The Journal of Clinical Endocrinology \& Metabolism, vol. 84, no. 2, pp. 561-566, 1999.

[17] J. G. Hollowell, N. W. Staehling, W. Dana Flanders et al., "T(4), and thyroid antibodies in the United States population (1988 to 1994): National Health and Nutrition Examination Survey (NHANES III)," The Journal of Clinical Endocrinology \& Metabolism, vol. 87, no. 2, pp. 489-499, 2002.

[18] K. Kasagi, N. Takahashi, G. Inoue, T. Honda, Y. Kawachi, and Y. Izumi, "Thyroid function in japanese adults as assessed by a general health checkup system in relation with thyroid-related antibodies and other clinical parameters," Thyroid, vol. 19, no. 9, pp. 937-944, 2009.

[19] P. Heydarian, A. Ordookhani, and F. Azizi, "Goiter rate, serum thyrotropin, thyroid autoantibodies and urinary iodine concentration in Tehranian adults before and after national salt iodization," Journal of Endocrinological Investigation, vol. 30, no. 5, pp. 404-410, 2007.

[20] F. Gołkowski, M. Buziak-Bereza, M. Trofimiuk, A. BałdysWaligórska, Z. Szybiński, and B. Huszno, "Increased prevalence of hyperthyroidism as an early and transient side-effect of implementing iodine prophylaxis," Public Health Nutrition, vol. 10, no. 8, pp. 799-802, 2007.

[21] S. Fountoulakis, G. Philippou, and A. Tsatsoulis, "The role of iodine in the evolution of thyroid disease in Greece: from endemic goiter to thyroid autoimmunity," Hormones, vol. 6, no. 1, pp. 25-35, 2007.

[22] K. Zaletel and S. Gaberšček, "Hashimoto's thyroiditis: from genes to the disease," Current Genomics, vol. 12, no. 8, pp. 576$588,2011$.

[23] G. J. Kahaly, H. P. Dienes, J. Beyer, and G. Hommel, "Iodide induces thyroid autoimmunity in patients with endemic goitre: a randomised, double-blind, placebo-controlled trial," European Journal of Endocrinology, vol. 139, no. 3, pp. 290-297, 1998.

[24] I. B. Pedersen, N. Knudsen, A. Carlé et al., "A cautious iodization programme bringing iodine intake to a low recommended level is associated with an increase in the prevalence of thyroid autoantibodies in the population," Clinical Endocrinology, vol. 75, no. 1, pp. 120-126, 2011.

[25] K. Aho, A. Gordin, K. Sievers, and J. Takala, "Thyroid autoimmunity in siblings: a population study," Acta Endocrinologica. Supplementum (Copenhagen), vol. 251, pp. 11-15, 1983.

[26] D. I. W. Phillips, D. C. Shields, J. M. Dugoujon, L. Prentice, P. McGuffin, and B. Rees Smith, "Complex segregation analysis of thyroid autoantibodies: are they inherited as an autosomal dominant trait?" Human Heredity, vol. 43, no. 3, pp. 141-146, 1993. 
[27] M. P. Desai and S. Karandikar, "Autoimmune thyroid disease in childhood: a study of children and their families," Indian Pediatrics, vol. 36, no. 7, pp. 659-668, 1999.

[28] M. Segni, J. Wood, I. Pucarelli, V. Toscano, R. Toscano, and A. M. Pasquino, "Clustering of autoimmune thyroid diseases in children and adolescents: a study of 66 families," Journal of Pediatric Endocrinology and Metabolism, vol. 14, no. 5, pp. 12711275, 2001.

[29] R. K. Marwaha, S. Sen, N. Tandon et al., "Familial aggregation of autoimmune thyroiditis in first-degree relatives of patients with juvenile autoimmune thyroid disease," Thyroid, vol. 13, no. 3, pp. 297-300, 2003.

[30] R. Villanueva, D. A. Greenberg, T. F. Davies, and Y. Tomer, "Sibling recurrence risk in autoimmune thyroid disease," Thyroid, vol. 13, no. 8, pp. 761-764, 2003.

[31] D. L. Mincer and I. Jialal, Thyroid, Hashimoto Thyroiditis, StatPearls Publishing, 2018. 


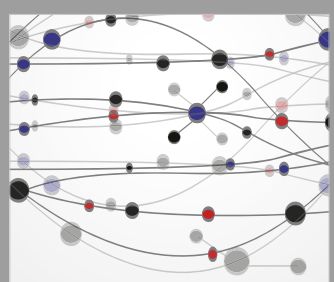

The Scientific World Journal
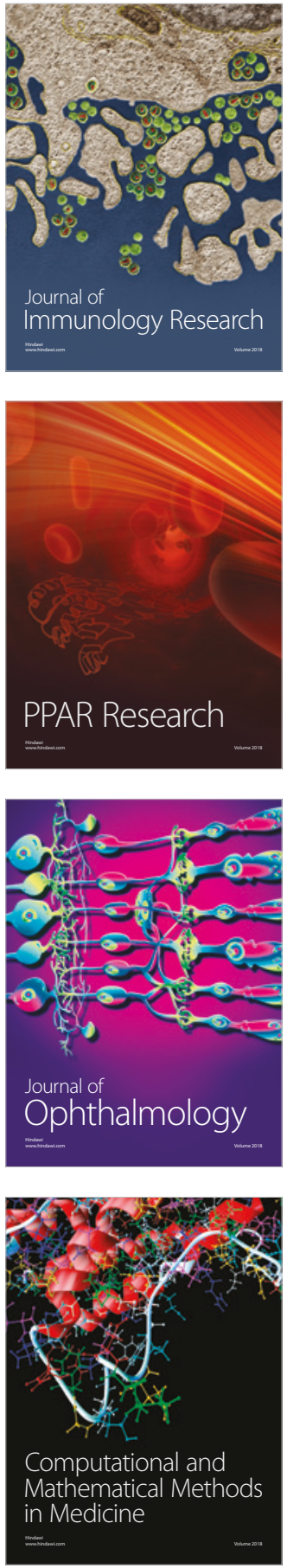

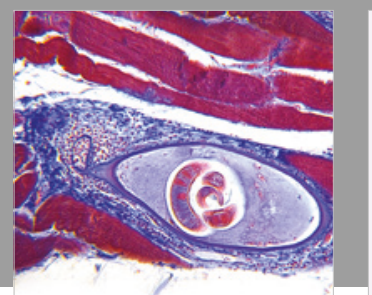

Gastroenterology Research and Practice

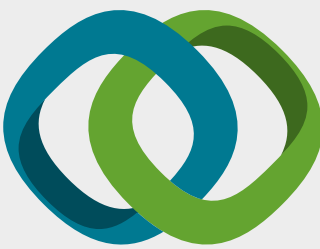

\section{Hindawi}

Submit your manuscripts at

www.hindawi.com
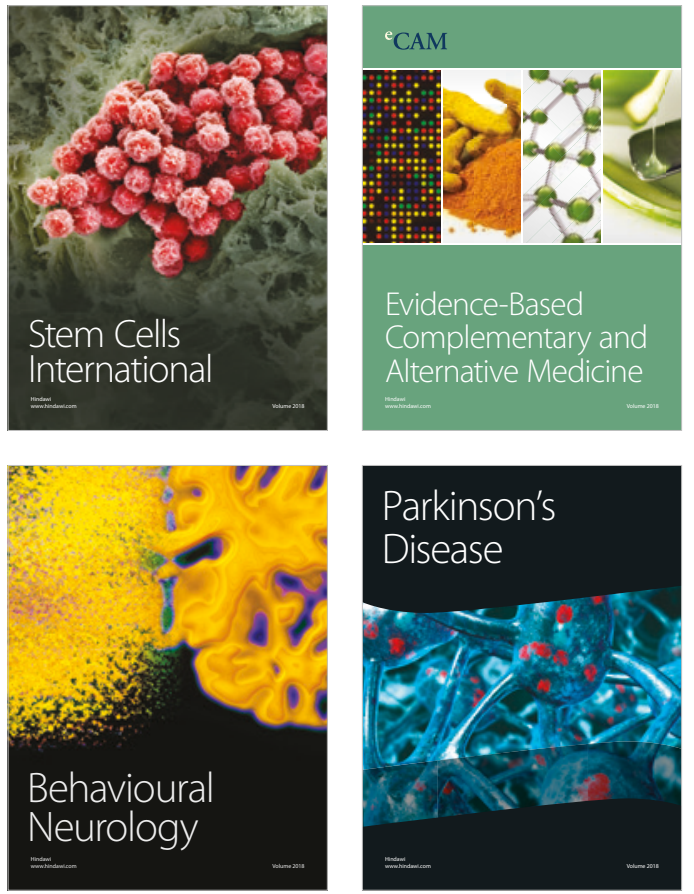

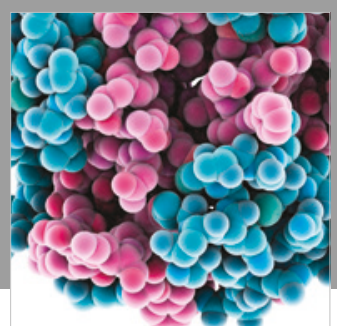

ournal of

Diabetes Research

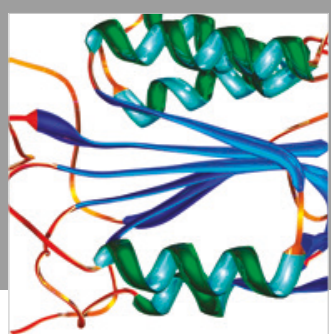

Disease Markers
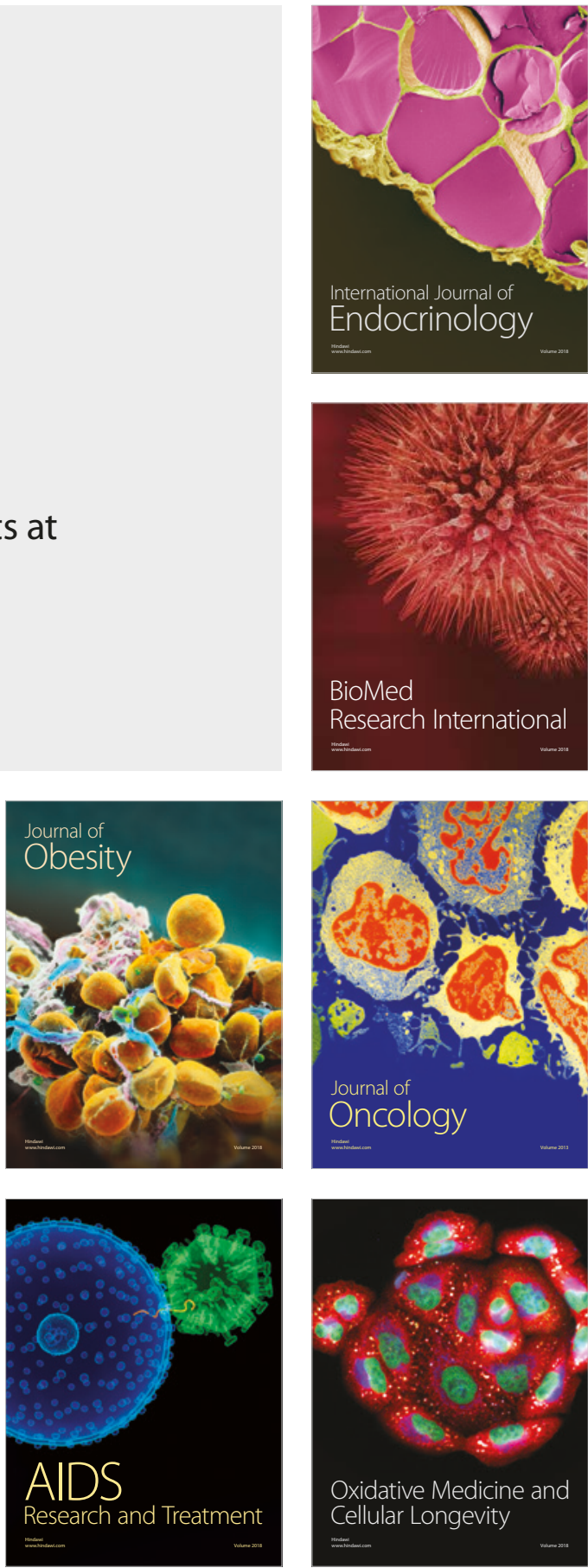